\title{
Importancia del seguimiento solar en las instalaciones fotovoltaicas
}

\author{
Importance of solar monitoring in photovoltaic installations
}

Importância do monitoramento solar em instalações fotovoltaicas

\section{ARTÍCULO ORIGINAL}

Denis Javier Aranguri Cayetano

daranguri@uns.edu.pe

https://orcid.org/0000-0002-6119-2072

Universidad Nacional del Santa, Ancash - Perú
Leandro Manuel Mariñes Delgado

manuxddd50@hotmail.com https://orcid.org/0000-0003-1626-9146

Universidad Nacional del Santa, Ancash - Perú
Romulo Augusto Castillo Banda

romulo castillobanda@hotmail.com https://orcid.org/0000-0003-4356-073X

Universidad Nacional del Santa, Ancash - Perú

Recibido 18 de Enero 2021 | Arbitrado y aceptado 10 de Marzo 2021 | Publicado en 05 Junio 2021

\section{RESUMEN}

El presente artículo consiste en determinar la cantidad de energía que no se aprovecha en un panel solar fijo con respecto a un panel solar movible (con seguimiento al sol). La toma de datos para esta investigación se llevó a cabo en las instalaciones de la Universidad Nacional del Santa, usando dos paneles idénticos, uno instalado en una posición fija y el otro con seguimiento solar variando su ángulo e inclinación horario, tomando en cuenta las condiciones climáticas de cada día (días claros y despejados). Se tomaron datos en los días 11, 12, 13, 19 de julio, 10,20, 26 de septiembre y $07,10,22,23,24,25,28$ de octubre. El día jueves 10 de octubre fue uno de los días con más horas de cielo despejado en total 9 hy 55 minutos, por lo que se usó como base para el análisis, el porcentaje de potencia no aprovechada fue mayor de 8:00 am a 10:00 am en un rango que va desde $13.71 \%$ hasta $47.63 \%$ y de $2: 00 \mathrm{pm}$ a $6: 00 \mathrm{pm}$ desde un $17.24 \%$ hasta $206.63 \%$ debido a que en dichas horas los rayos del sol inciden de forma inclinada.

Palabras Clave: Ángulo horario, Inclinación, potencia desaprovechada, seguimiento solar.

\begin{abstract}
This article consists of determining the amount of energy that is not used in a fixed solar panel with respect to a movable solar panel (with sun tracking). Data collection for this research was carried out at the facilities of the National University of Santa, using two identical panels, one installed in a fixed position and the other with solar tracking varying its angle and hourly inclination, taking into account the conditions. weather conditions for each day (clear and clear days). Data were collected on July $11,12,13,19$, September 10, 20, 26, and October 7, 10, $22,23,24,25,28$. Thursday, October 10 , was one of the days with more hours of clear skies in total $9 \mathrm{~h}$ and 55 minutes, so it was used as a basis for the analysis, the percentage of unused power was greater from 8:00 a.m. to 10:00 a.m. : 00 am in a range that goes from $13.71 \%$ to $47.63 \%$ and from $2: 00 \mathrm{pm}$ to $6: 00 \mathrm{pm}$ from $17.24 \%$ to $206.63 \%$ because in those hours the sun's rays affect in an inclined way.
\end{abstract}

Keywords: Hour angle, Inclination, wasted power, solar tracking.

\section{RESUMO}

Este artigo consiste em determinar a quantidade de energia que não é utilizada em um painel solar fixo em relação a um painel solar móvel (com rastreamento solar). A coleta de dados para esta pesquisa foi realizada nas instalações da Universidade Nacional de Santa, utilizando dois painéis idênticos, um instalado em posição fixa e outro com rastreamento solar variando seu ângulo e inclinação horária, levando em consideração as condições climáticas. para cada dia (dias claros e claros). Os dados foram coletados em 11, 12, 13, 19 de julho, $10,20,26$ de setembro e $7,10,22,23$, 24, 25, 28 de setembro. Quinta-feira, 10 de outubro, foi um dos dias com mais horas de céu claro no total de 9 he 55 minutos, por isso foi usado como base para a análise, o percentual de energia não utilizada foi maior das 8h00 às 10h00: 00h00: 00h00 em uma faixa que vai de $13,71 \%$ a $47,63 \%$ e das $14 \mathrm{~h} 00$ às $18 \mathrm{~h} 00$ de $17,24 \%$ a $206,63 \%$ porque nessas horas os raios solares atuam de forma inclinada.

Palavras-chave: Ângulo horário, Inclinação, energia desperdiçada, rastreamento solar. 


\section{INTRODUCCION}

El uso de las energías renovable es cada vez más común, entre ellas una de las más destacadas es la energía Solar (Hilcu, 2021), no obstante, el mayor problema de la energía solar es su eficiencia. Uno de los factores que afecta la eficiencia de un panel solar es que a la hora de su instalación, se coloca fijo en una sola posición y no siempre está orientado directamente a los rayos del Sol (Izquierdo y Pacheco, 2017), haciendo que no pueda aprovechar la radiación solar en su totalidad, por otra parte, en las instalaciones con seguimiento solar el movimiento sincronizado entre el panel solar y la posición aparente de sol durante el día y a través del año, permite aprovechar una mayor cantidad de radiación incidente, esto aumentará los niveles de corriente y voltaje producidos por el panel generando una mayor potencia, dándole así, un mejor rendimiento a comparación de un panel fijo.

A pesar de que la opción de usar una instalación con seguimiento solar sería la más adecuada para evitar este desaprovechamiento de potencia en los paneles fijos, actualmente no es muy rentable debido a su elevado costo de inversión.

\section{ANTECEDENTES}

-Sandoval (2020) en el artículo "Arreglos fotovoltaicos inteligentes con modelo LFSR-reconfigurable" donde se revisaron técnicas de síntesis de celdas fotoelectroquímica, factores de optimización en arreglos fotovoltaicos y electrónica para la definición de capas funcionales de separación espectral, concentración solar, conversión fotoeléctrica, registro de energía, mecanismos de seguimiento solar y recuperación de calor regenerativo, adaptados a través de redes neuronales aplicadas.

-Echenique et al. (2018) en el artículo "Sistema de seguimiento solar para paneles fotovoltaicos" que desarrolló un seguidor solar con dos ejes para ser aplicado a panales fotovoltaicos de tal forma que se demuestra un incremente en la producción de los paneles de 20 a 30\%, dependiendo del mes y la hora del día.

•Camacás, P (2017) en el artículo científico "Diseño e implementación de un sistema automático de seguimiento solar para un generador termo solar" nos explica la implementación de un sistema basado en ecuaciones de posicionamiento astronómico que permitirán saber la posición del sol en cada hora del día, el sistema de automatización se diseñó y programo utilizando Arduino como el controlador principal. 
-Arreola, G (2015) en el artículo científico "Diseño, construcción y evaluación” nos dice que, utilizando un colector solar con seguimiento, la energía total recibida en un día claro puede ser del orden de un 30-45\% mayor que para el mismo colector solar estático.

-Lusson, C (2015) en el artículo científico "Seguidor Solar, optimizando el aprovechamiento de la energía solar" nos habla de un de un dispositivo encargado de obtener las coordenadas del Sol en cualquier momento del día, es decir, un seguidor solar a dos ejes por punto luminoso.

-Arreola, R. et al. (2015) en el artículo científico “Diseño, construcción y evaluación de un sistema de seguimiento solar para un panel fotovoltaico" habla sobre el desarrollo de un sistema de seguimiento autónomo y capaz de seguir el movimiento de sol, acoplándose a un panel fotovoltaico para mejorar la eficiencia en la captura de energía.

•Abal, G \& Durañona, V (2013) en el artículo científico "Manuel Técnico de Energía Solar Térmica" tiene como objetivo reunir en una publicación los conceptos básicos, recomendaciones (buenas practicas) y otras informaciones útiles para desempeñarse como Responsable Técnico de Instalaciones (RTI) de Energía Solar Térmica (EST) en el Uruguay.

\section{METODOLOGÍA}

La investigación es de tipo experimental cuantitativa, se usó dos paneles fotovoltaicos de 50 watts (en el mes de julio) y dos paneles de 150 watts (de septiembre en adelante); uno será un panel fijo y el otro tendrá seguimiento al sol, este último estará apoyado en un soporte movible con dos ejes de movimiento: el movimiento horario y la variación de su inclinación (López, A. G. 1985). Con los paneles trabajando en paralelo obtendremos datos de corriente y voltaje, registrando los valores cada cinco minutos. En el caso del panel con seguimiento solar, se ajustará a un ángulo de inclinación optimo según el mes en que se realice el experimento, y a lo largo de cada día se ajustará el ángulo horario de tal manera que cada hora tenga su respectivo ángulo horario, la ecuación del tiempo nos permitirá determinar el tiempo solar verdadero. Se pretende medir tres días a la semana desde las ocho de la mañana hasta las seis de la tarde, siempre y cuando el día cumpla con las condiciones óptimas requeridas para la investigación (día despejado).

\subsection{CRITERIOS PARA LA TOMA DE DATOS}

Al momento de medir se tendrán los siguientes criterios:

- Registrar la irradiancia. 
- Tomar el dato de corriente en ambos paneles, siempre y cuando la irradiancia se encuentre lo más cercana posible al valor que se registró primero ya que esta es muy variable.

- Tomar el valor de voltaje en los paneles, este se toma al último ya que es poco sensible al cambio de irradiación, pero de igual modo procurando que el valor de irradiancia sea similar al que se registró primero.

\subsection{PARÁMETROS DE LOS PANELES:}

Panel fijo. Para el panel fijo se consideró un ángulo horario de cero grados $\left(\omega=0^{\circ}\right) \mathrm{y}$ una inclinación de veinte grados sexagesimales $\left(\beta=20^{\circ}\right)$, ángulo recomendado para las instalaciones fotovoltaicas en la ciudad de Chimbote.

Ángulo medio. El ángulo horario se varía $15^{\circ}$ en un intervalo de una hora (Sumano, F, 2012), pero en esta investigación tomaremos nuevos ángulos. Los datos se tomarán cada 5 minutos, se ha considerado conveniente usar "ángulos medios" $\left(\omega^{*}\right)$ de los ángulos tradicionales $(\omega)$, todo esto con la finalidad de obtener mayor aprovechamiento de la radiación y obtener mejores lecturas de corriente y voltaje (Escobar, M. 2010).

\section{TABLA I}

Ángulos horarios medios por cada intervalo de hora $\left({ }^{\circ}\right)$

\begin{tabular}{cc}
\hline $\begin{array}{l}\text { Intervalo } \\
\text { horario }\end{array}$ & $\begin{array}{l}\text { Ángulo } \\
\left(\omega^{*}\right)\end{array}$ \\
\hline 7:00 - 7:55 & -67.5 \\
8:00 - 8:55 & -52.5 \\
9:00 - 9:55 & -37.5 \\
10:00 - 10:55 & -22.5 \\
$11: 00-11: 55$ & -7.5 \\
$12: 00-12: 55$ & 7.5 \\
$13: 00-13: 55$ & 22.5 \\
$14: 00-14: 55$ & 37.5 \\
$15: 00-15: 55$ & 52.5 \\
$16: 00-16: 55$ & 67.5 \\
$17: 00-17: 55$ & 82.5
\end{tabular}


Nota: Estos ángulos son la semisuma de los ángulos horarios tradicionales $0^{\circ}, 15^{\circ}, 30^{\circ}$, $45^{\circ}$, etc. cada uno con su respectivo intervalo horario.

Inclinación ( $\boldsymbol{\beta})$. La inclinación óptima que se usará para cada mes, tiene la finalidad de permitir al panel móvil aprovechar la mayor cantidad de irradiación incidente. (Celso, R. 2015)

TABLA 11

Radiación Solar global estimada con distintos ángulos de inclinación para la ciudad de Chimbote

\begin{tabular}{lllllllllllll}
\hline MES & Ene & Febr & Mar & Abril & May & Juni & Julio & Ago & Setie & Octu & Nov & Dic \\
\hline $\boldsymbol{\beta}\left({ }^{\circ}\right)$ & \multicolumn{10}{c}{ HT $(\mathbf{k W} / \mathbf{m} 2)$} \\
\hline $\mathbf{0}$ & 0.482 & 0.505 & 0.712 & 0.649 & 0.553 & 0.538 & 0.486 & 0.520 & 0.457 & 0.517 & 0.410 & 0.653 \\
$\mathbf{5}$ & 0.471 & 0.498 & 0.713 & 0.664 & 0.575 & 0.565 & 0.507 & 0.535 & 0.460 & 0.513 & 0.403 & 0.634 \\
$\mathbf{1 0}$ & 0.458 & 0.489 & 0.711 & 0.674 & 0.594 & 0.588 & 0.525 & 0.547 & 0.462 & 0.506 & 0.394 & 0.611 \\
$\mathbf{1 5}$ & 0.442 & 0.478 & 0.704 & 0.681 & 0.609 & 0.607 & 0.539 & 0.555 & 0.461 & 0.496 & 0.383 & 0.585 \\
$\mathbf{2 0}$ & 0.425 & 0.464 & 0.692 & 0.683 & 0.620 & 0.623 & 0.551 & 0.560 & 0.457 & 0.484 & 0.370 & 0.556 \\
$\mathbf{2 5}$ & 0.405 & 0.447 & 0.677 & 0.681 & 0.627 & 0.635 & 0.559 & 0.562 & 0.450 & 0.469 & 0.356 & 0.525 \\
$\mathbf{3 0}$ & 0.384 & 0.428 & 0.657 & 0.675 & 0.630 & 0.643 & 0.563 & 0.560 & 0.442 & 0.452 & 0.340 & 0.491 \\
$\mathbf{3 5}$ & 0.361 & 0.407 & 0.634 & 0.664 & 0.629 & 0.646 & 0.564 & 0.555 & 0.430 & 0.432 & 0.322 & 0.455 \\
$\mathbf{4 0}$ & 0.337 & 0.385 & 0.606 & 0.650 & 0.625 & 0.646 & 0.562 & 0.546 & 0.417 & 0.410 & 0.303 & 0.418 \\
$\mathbf{4 5}$ & 0.311 & 0.360 & 0.575 & 0.631 & 0.616 & 0.641 & 0.556 & 0.535 & 0.401 & 0.386 & 0.284 & 0.379 \\
$\mathbf{5 0}$ & 0.285 & 0.334 & 0.541 & 0.609 & 0.604 & 0.633 & 0.547 & 0.520 & 0.383 & 0.360 & 0.263 & 0.340 \\
\hline
\end{tabular}

Nota: Este cuadro se basa en el análisis estadístico de la irradiación solar mensual sobre superficies con diferentes ángulos de inclinación, por lo que proporciona la inclinación óptima en la que se aprovecharía la máxima irradiación.

Irradiación $\left(\mathbf{W} / \mathbf{m}^{2}\right)$. Esta es la responsable de que se genere concentración de energía en ambos paneles, su incidencia en la superficie de la tierra depende de las condiciones de cada día: si está despejado habrá más irradiación incidente que cuando está nublado (Lorente, J., 2010).

Ecuación del tiempo (minutos). Es la discrepancia entre el movimiento del Sol medio (perfectamente uniforme con intervalos de 24 horas entre dos pasos consecutivos del Sol por el meridiano local) y el movimiento aparente del Sol verdadero, en minutos (Duffie, J. A. and Beckman, W. A., 1991): 
$E_{t}=229.2(0.000075+0.001868 \cos B-0.032077 \sin B$

$-0.014615 \cos 2 B-0.04089 \sin 2 B)$,

$B=\frac{360}{365}(n-1)$,

$\mathrm{E}_{\mathrm{t}}=$ Ecuación del tiempo

$\mathrm{B}=$ Factor de corrección

$\mathrm{n}=$ Número de orden de día en el año

\subsection{POTENCIAS EN AMBOS PANELES}

Con la corriente de corto circuito y el voltaje en circuito abierto se puede generar potencia nominal en ambos paneles, la variable que se usará para la comparación de ambos paneles en la investigación.

P.MED $($ Watts $)=$ Corriente $(A) *$ Voltaje $(V)$,

P.MED = Potencia Nominal puntual medida

\subsection{PORCENTAJE COMPARATIVO}

Una vez obtenidas las potencias de ambos paneles se calcula un porcentaje comparativo poniendo como base la potencia del panel con seguimiento solar, ya que se desea demostrar que es más provechoso que un panel fijo. El porcentaje de potencia se evaluará por medición puntual para analizar con una gráfica el comportamiento del porcentaje de potencia que no se aprovecha en las instalaciones fijas durante el día.

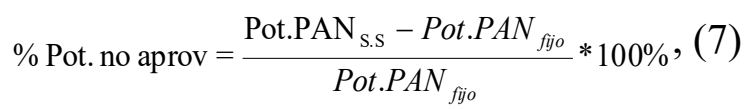

$\%$ Pot. no aprov $=$ Porcentaje de potencia no aprovechada 


\section{MATERIALES}

Fig. 1. Dos multímetros

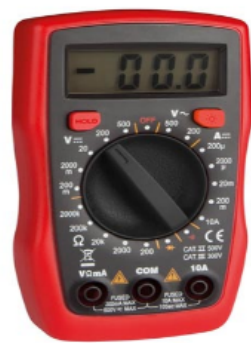

Fig. 2. Radiómetro

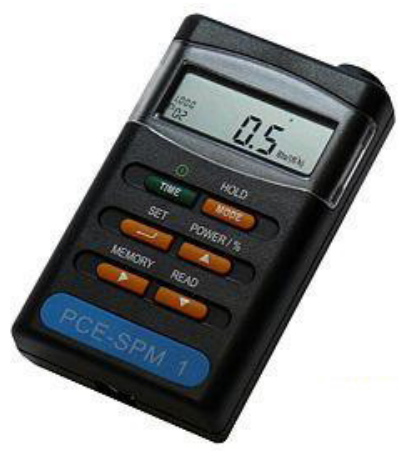

Fig. 3. Inclinómetro

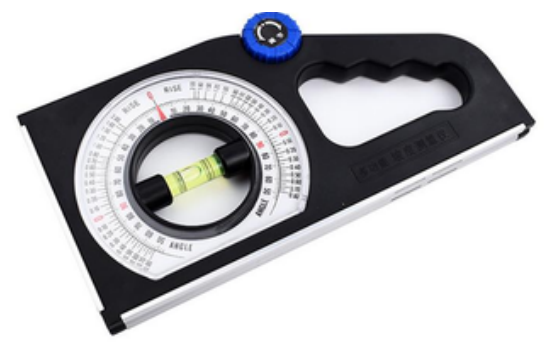


Fig. 4. Dos paneles policristalinos

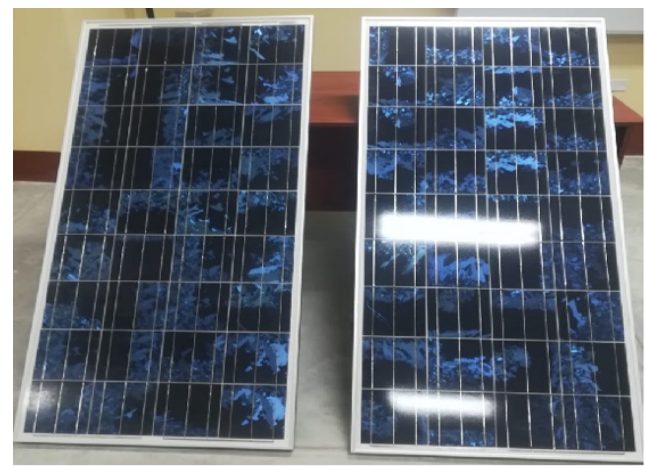

Fig. 5. Un soporte con los dos movimientos solares de inclinación y ángulo horario.

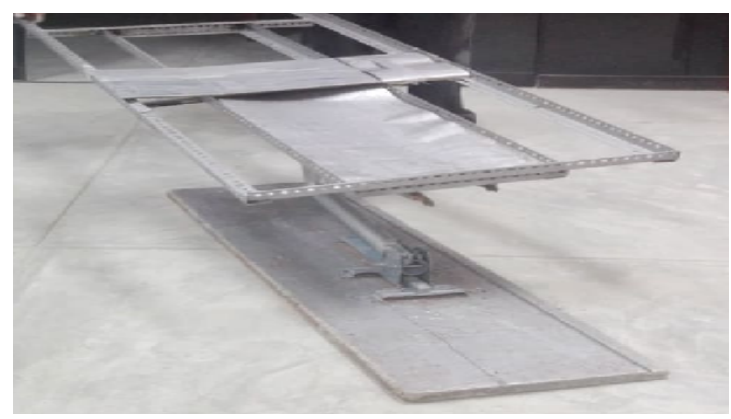

\section{PROCEDIMIENTO}

La toma de datos será desde las ocho de la mañana hasta las seis de la tarde en las instalaciones de la Universidad Nacional del Santa con dos paneles gemelos, no obstante, solo se analizará los datos registrados en las horas de cielo despejado. Uno de los paneles estará fijo en una sola posición y el otro con seguimiento solar variando su inclinación y su ángulo horario, ambos estarán orientados al norte. Cada medición se hará en un intervalo de cinco minutos midiendo paralelamente los datos de radiación, corriente y voltaje en ambos paneles con los multímetros, para determinar el porcentaje de potencia desaprovechada en el panel estático con respecto al panel móvil. Como se sabe hay ángulos horarios para cada hora, pero para aprovechar al máximo la radiación en el panel con seguimiento solar, se optará por usar "ángulos medios horarios", estos valores serán las semisumas de los ángulos tradicionales (ver TABLA 1).

Con la ecuación del tiempo se determinará el desfase horario y añadiéndole este tiempo al intervalo de horas se variarán los ángulos medios midiéndolos con en inclinómetro. 


\subsection{Ecuación del tiempo}

Se determinar el desfase horario para el día 10 de octubre:

\section{Usando la ecuación (2):}

$\mathbf{n}_{\text {(día al año) }}=283$

$B=\frac{360(283-1)}{365}=278.137$

\section{Usando la ecuación (1):}

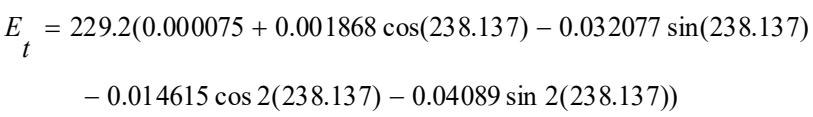

$E_{t}=13.197 \mathrm{~min} \approx 13 \mathrm{~min}$

Los valores resultantes de la ecuación del tiempo en minutos se redondean al valor inferior inmediato en minutos que sea múltiplo de cinco, debido a que las mediciones son cada 5 minutos desde las 8 de la mañana hasta las 6 de la tarde. Entonces:

$$
E_{t}=13 \mathrm{~min} \approx 10 \mathrm{~min}
$$

Ahora sumamos este retraso a la hora inicial y a la hora final de un intervalo horario de la Tabla I, para determinar el verdadero intervalo horario, conservando el ángulo original.

Tomaremos como ejemplo el intervalo horario del ángulo -52.5: (8:00-8:55) am 
Para el día 10 de octubre entonces se determinó con ayuda de la ecuación del tiempo el verdadero intervalo horario.

$$
\begin{aligned}
& h i^{*}=8: 00+0: 10=8: 10 \mathrm{am} \\
& h f^{*}=8: 55+0: 10=9: 05 \mathrm{am}
\end{aligned}
$$

hi: Inicio del primer intervalo de toma de datos corregida.

hf: Término del primer intervalo de toma de datos corregida.

Todos los intervalos horarios del 10 de octubre se verán afectados por éste retraso horario.

Contando con la inclinación óptima para cada mes y el desfase horario para cada día se tienen los parámetros necesarios para proceder a medir los valores de corriente y voltaje en el panel con seguimiento solar.

Por cada medición, se ingresan los valores de radiación, corriente y voltaje a Excel.

\section{1}

\section{CONSIDERACIONES}

Para nuestra investigación el día es óptimo cuando está despejado, porque al ver radiación directa el seguimiento al sol es fundamental para aprovechar la radiación al máximo. Sin embargo, cuando el día esta nublado será el panel fijo el que genere mejores valores debido a la radiación difusa. Además, frecuentemente ocurre que la captación de energía disminuye debido al incremento de temperatura en las celdas del panel, lo que ocasiona una disminución en el voltaje, entre otros factores como la velocidad del viento. (Arreola.G,2015)

\section{ANÁLISIS DE RESULTADOS}

A continuación, se pondrá como ejemplo las mediciones del día 10 de octubre.

Inclinación y ángulo horario del Panel fijo. Como se detalló en un inicio, el panel fijo tendrá una inclinación de $20^{\circ}$ y un ángulo horario de $0^{\circ}$. 
Inclinación de Panel con seguimiento Solar. Para el mes de octubre, el ángulo de inclinación optimo es $0^{\circ}$ (ver TABLA 11) pero se optó por usar un ángulo de inclinación $10^{\circ}$, para no salir del plano real en el cual se considera que las instalaciones fotovoltaicas deben tener un cierto grado de inclinación obligatoria para evitar que se almacene el agua de las lluvias.

TABLA 3

\begin{tabular}{|c|c|c|c|c|c|c|c|c|c|c|}
\hline \multirow{3}{*}{ HORA } & \multirow{3}{*}{$\omega^{*}$} & \multicolumn{4}{|c|}{ PANEL CON } & & & & \multirow{3}{*}{$\begin{array}{c}\text { POTENCIA } \\
\text { NO } \\
\text { APROVE.(W) }\end{array}$} & \multirow{3}{*}{$\begin{array}{c}\% \text { DE } \\
\text { POTENCIA NO } \\
\text { APROVECHADA }\end{array}$} \\
\hline & & \multicolumn{3}{|c|}{ SEGUIMIENTO } & \multirow{2}{*}{$\begin{array}{l}\text { IRRADIACIÓN } \\
(\mathrm{W} / \mathrm{m} 2)\end{array}$} & \multicolumn{3}{|c|}{ PANEL FIJO } & & \\
\hline & & $\overline{\mathrm{I}(\mathrm{A})}$ & $\mathrm{V}(\mathrm{V})$ & $\mathrm{P}(\mathrm{W})$ & & $\mathrm{I}(\mathrm{A})$ & $\mathrm{V}(\mathrm{V})$ & $\mathrm{P}(\mathrm{W})$ & & \\
\hline $08: 00$ & & 2.98 & 20.4 & 60.792 & 398.5 & 2.2 & 18.9 & 41.58 & 19.212 & $46.20 \%$ \\
\hline 08:05 & - & 1.71 & 20.3 & 34.713 & 270.1 & 1.3 & 18.9 & 24.57 & 10.143 & $41.28 \%$ \\
\hline $08: 10$ & $67.5^{\circ}$ & 1.85 & 19.9 & 36.815 & 277.3 & 1.35 & 19.3 & 26.055 & 10.76 & $41.30 \%$ \\
\hline $08: 15$ & & 2.76 & 19.4 & 53.544 & 137.2 & 2.08 & 19.3 & 40.144 & 13.4 & $33.38 \%$ \\
\hline $08: 20$ & & 2.41 & 19.8 & 47.718 & 410.5 & 1.87 & 19.3 & 36.091 & 11.627 & $32.22 \%$ \\
\hline $08: 25$ & & 2.99 & 20.7 & 61.893 & 496.8 & 2.15 & 19.5 & 41.925 & 19.968 & $47.63 \%$ \\
\hline $08: 30$ & & 2.72 & 19.8 & 53.856 & 484.7 & 1.98 & 19.5 & 38.61 & 15.246 & $39.49 \%$ \\
\hline $08: 35$ & & 2.93 & 19.9 & 58.307 & 389.5 & 2.23 & 19.5 & 43.485 & 14.822 & $34.09 \%$ \\
\hline $08: 40$ & & 3.78 & 19.9 & 75.222 & 470.3 & 3.1 & 19.8 & 61.38 & 13.842 & $22.55 \%$ \\
\hline $08: 45$ & & 3.96 & 19.8 & 78.408 & 488.6 & 3.35 & 19.9 & 66.665 & 11.743 & $17.61 \%$ \\
\hline $08: 50$ & $525^{\circ}$ & 4.35 & 20.2 & 87.87 & 489.1 & 4.03 & 20 & 80.6 & 7.27 & $9.02 \%$ \\
\hline $08: 55$ & J2.J & 4.75 & 19.7 & 93.575 & 517.8 & 4.12 & 20 & 82.4 & 11.175 & $13.56 \%$ \\
\hline 09:00 & & 5.25 & 20.10 & 105.525 & 562.3 & 4.40 & 19.1 & 84.0 & 21.485 & $25.57 \%$ \\
\hline 09:05 & & 8.70 & 20.20 & 175.740 & 845.6 & 6.88 & 20.1 & 138.3 & 37.452 & $27.08 \%$ \\
\hline 09:10 & & 8.85 & 20.50 & 181.425 & 867.3 & 6.93 & 20.1 & 139.3 & 42.132 & $30.25 \%$ \\
\hline 09:15 & & 8.98 & 19.20 & 172.416 & 878.4 & 7.43 & 19.1 & 141.9 & 30.503 & $21.49 \%$ \\
\hline $09: 20$ & & 8.38 & 20.50 & 171.688 & 823.4 & 6.93 & 20.1 & 139.2 & 32.495 & $23.35 \%$ \\
\hline $09: 25$ & & 8.15 & 20.60 & 167.890 & 803.4 & 6.87 & 20.3 & 139.5 & 28.429 & $20.38 \%$ \\
\hline 09:30 & & 8.85 & 20.70 & 183.195 & 865.7 & 7.42 & 20.2 & 149.9 & 33.311 & $22.22 \%$ \\
\hline $09: 35$ & & 8.73 & 20.60 & 179.735 & 845.6 & 6.67 & 20 & 133.4 & 46.335 & $34.73 \%$ \\
\hline 09:40 & & 8.43 & 20.40 & 171.870 & 818.9 & 7.10 & 19.8 & 140.6 & 31.29 & $22.26 \%$ \\
\hline $09: 45$ & & 8.35 & 20.10 & 167.835 & 810.3 & 7.10 & 19.6 & 139.2 & 28.675 & $20.61 \%$ \\
\hline 09:50 & $375^{\circ}$ & 8.28 & 20.20 & 167.256 & 806.4 & 7.08 & 19.6 & 138.8 & 28.488 & $20.53 \%$ \\
\hline $09: 55$ & & 8.20 & 20.00 & 164.000 & 800.9 & 7.03 & 19.5 & 137.1 & 26.915 & $19.63 \%$ \\
\hline $10: 00$ & & 8.25 & 20.10 & 165.825 & 805.4 & 7.08 & 19.6 & 138.7 & 27.155 & $19.58 \%$ \\
\hline $10: 05$ & & 8.10 & 19.90 & 161.190 & 794.5 & 7.05 & 19.5 & 137.5 & 23.715 & $17.25 \%$ \\
\hline $10: 10$ & & 8.25 & 20.10 & 165.825 & 807.6 & 7.23 & 19.6 & 141.6 & 24.215 & $17.10 \%$ \\
\hline $10: 15$ & & 8.13 & 20.20 & 164.125 & 796.3 & 7.20 & 19.6 & 141.1 & 23.005 & $16.30 \%$ \\
\hline
\end{tabular}

Mediciones puntuales de corriente, voltaje potencia e irradiación, del día 10/10/19 con dos paneles gemelos de $150 \mathrm{~W}$.

Nota: Se registraron las lecturas de corriente y voltaje que generaron ambos paneles al mismo tiempo bajo las mismas condiciones, la toma de datos fue durante 10 horas desde 
las 8:00 am hasta las 6:00 pm pero para el análisis solo se mostrará las 3 primeras horas, como podemos observar en el cuadro, los valores de corriente en el panel móvil son superiores al panel fijo, y el porcentaje de potencia no aprovechada en el panel fijo llegó hasta un $47.63 \%$ en ese intervalo de horas, dicho porcentaje va disminuyendo conforme nos acerquemos al medio día ya que el ángulo horario de ambos paneles se empieza a igualar, y la potencia extra generada en el panel móvil solo se da gracias al ángulo de inclinación óptimo para dicho mes, cabe resaltar que al alejarnos del medio día el porcentaje de potencia desaprovechada vuelve a incrementar como si aprecia en la Fig.3 y Fig4 .1

\subsection{ANÁLISIS DEL PORCENTAJE DE POTENCIA NO APROVECHADA EN}

\section{EL PANEL FIJO}

Empleando la ecuación (11) podemos obtener el porcentaje de potencia desaprovechada en el panel fijo respecto al panel con seguimiento solar, viendo su comportamiento en función del tiempo.

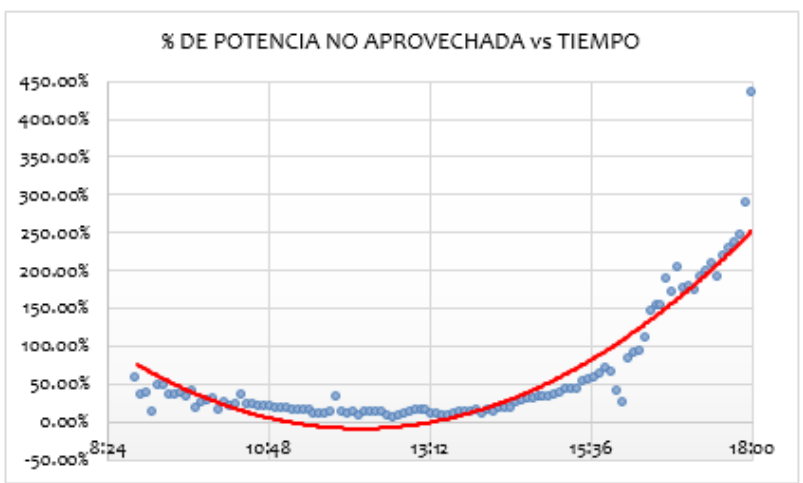

Fig. 3- Porcentaje de potencia no aprovechada durante un día de medición desde las 8:00 am hasta las 5:55 pm para el día 10/10/19. 
Denis Javier Aranguri Cayetano, Leandro Manuel Mariñes Delgado, Romulo Augusto Castillo Banda

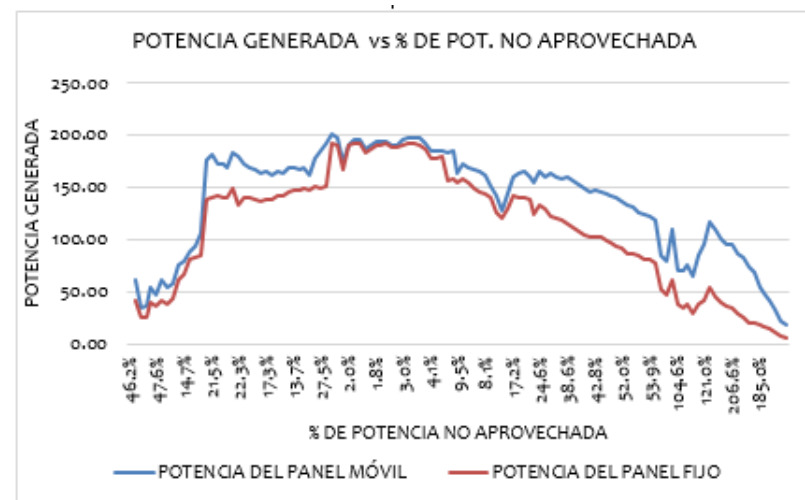

Fig. 4. Porcentaje de potencia no aprovechada en el panel fijo con respecto al panel móvil 10/10/19

En la Fig. 3 y Fig. 4 se observa el comportamiento que tiene el \% de potencia no aprovechada en el panel fijo respecto al panel con seguimiento solar. Esta gráfica demuestra que el desaprovechamiento es mayor de 8:00 am a 10:00 am en un rango que va desde $13.71 \%$ hasta $47.63 \%$ y de $2: 00 \mathrm{pm}$ a $6: 00 \mathrm{pm}$ desde un $17.24 \%$ hasta 206.63 $\%$ debido a que en dichas horas los rayos del sol inciden de forma inclinada sobre la superficie horizontal del panel fijo y de forma directa sobre el panel con seguimiento solar.

\subsection{ANÁLISIS DE LA POTENCIA GENERADA POR AMBOS PANELES SOMETIDOS A DISTINTOS NIVELES DE IRRADIACIÓN}

También se puede determinar el comportamiento que tiene la potencia nominal de ambos paneles a distintos niveles de irradiancia, tomando lecturas de irradiación con un radiómetro en un plano horizontal.

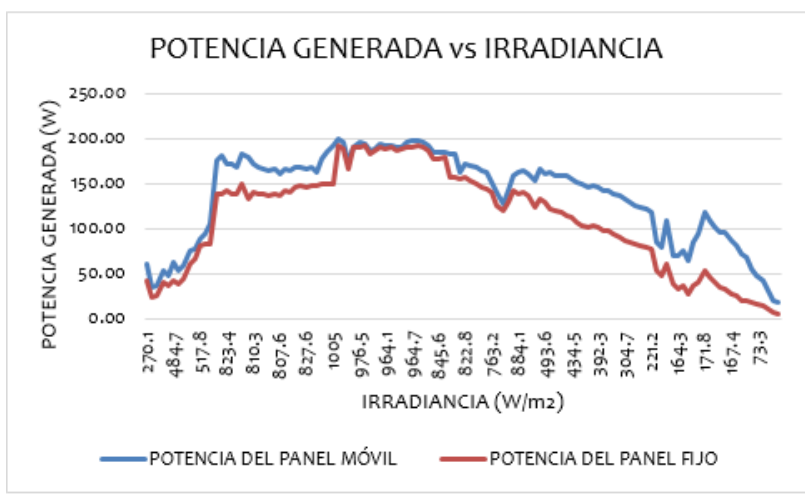

alejadas del medio día.
En la Fig. 5 se observa cómo es que a igual irradiancia solar, el panel móvil genera mayor potencia que el panel fijo, aumentando la diferencia de potencia generada en las horas

Fig. 5- Potencia generada a distintos niveles de irradiancia del 10/10/19. 
TABLA IV

Cuadro resumen de potencias y $\%$ de un día medido (10/10/19)

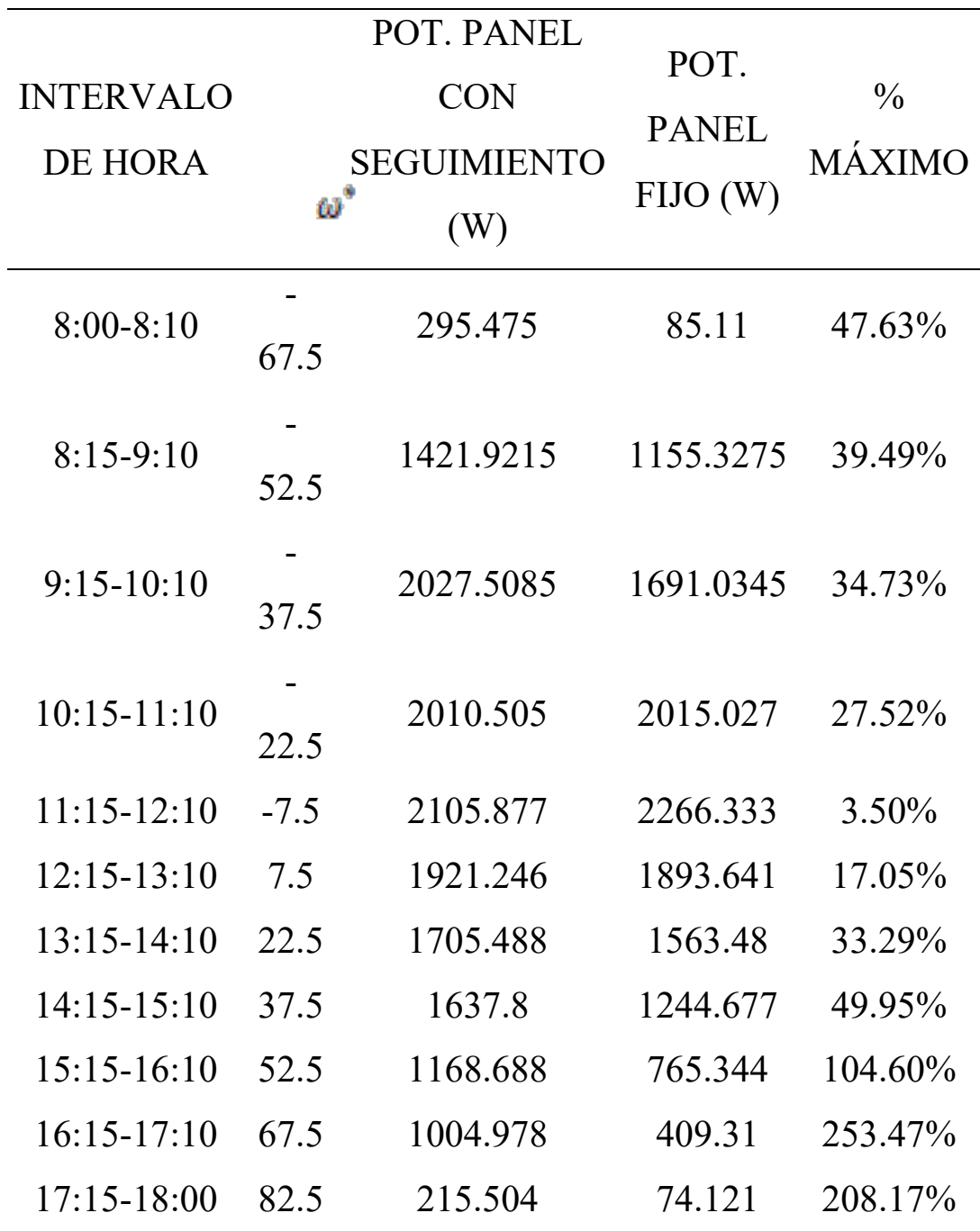

Nota: Se observa el máximo porcentaje de potencia no aprovechada que se dio en cada hora de medición, el durante el día se presenta mayores porcentajes de desaprovechamiento de 8:00 am a 10:00 am y de 2:00 pm a 6:00 pm.

\section{CONCLUSIONES}

- El porcentaje de energía no aprovechada promedio en los días medidos es $28.31 \%$.

- Equiparar costos por generación de energía, tomando precios facturados.

- Proponer alternativas para mecanismos y/o sistemas de seguimiento solar.

\section{REFERENCIAS}

[1] López, A. G., Sistemas de seguimiento del sol., Segunda (Ed.). Barcelona, Marcombo Boixareu Editores, 1985, p. 6. 
[2] Sumano, F. El ángulo horario es igual a cero al medio día solar y adquiere un valor de $15^{\circ}$ de longitud. 2012, p. 15.

[3] Lorente, J. Una parte de la irradiancia solar que incide en el suelo proviene directamente del disco solar (irradiancia solar directa) y otra proviene del cielo y las nubes (irradiancia solar difusa).2010, p 5.

[4] Duffie J. A. and Beckman. La Education del tiempo.1991, p 11

[5] Hilcu, M. El impacto positivo de las energías renovables sobre el medioambiente. 2021.

[6] Izquierdo, I., Pacheco, M. Evaluación de la eficiencia de paneles solares como sistema de captación de energía para edificaciones del área urbana de Cuenca. 2019. 